Journal of Thermal Engineering, Vol. 6, No. 1, pp. 170-186, January, 2020

Yildiz Technical University Press, Istanbul, Turkey

\title{
EFFECT OF MAGNETIC FIELD ORIENTATION ON NANOFLUID FREE CONVECTION IN A POROUS CAVITY: A HEAT VISUALIZATION STUDY
}

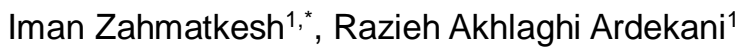

\begin{abstract}
Effect of magnetic field orientation on free convection of several water-based nanofluids in a square porous cavity is analyzed in this study. To this aim, the heatline visualization technique is implemented for the first time. Moreover, streamlines and isotherms are employed to present fluid flow and temperature distribution. The governing equations are transformed into a dimensionless form and then solved using the finite-volume method. Computations are undertaken for different orientations and magnitudes of the imposed magnetic field in circumstances with distinct Rayleigh numbers and the nanoparticles types and volume fractions. The corresponding results are presented in terms of dimensionless distributions of streamlines, isotherms, and heatlines as well as numerical values of the flow strength and the average Nusselt number. Inspection of the results demonstrates that increase in the magnetic field strength deteriorates the heat transfer rate. This effect, however, diminishes with rise in the magnetic field inclination angle.
\end{abstract}

Keywords: Magnetohydrodynamics, Heatline, Porous Media, Nanofluid, Cavity

\section{INTRODUCTION}

Magnetohydrodynamic (MHD) free convection of electrically conducting fluids in cavities has attracted the interest of researchers in the past due to possible applications in many industrial and technological fields such as metal casting, fusion reactors, and crystal growth. It was found that, as a result of the imposed magnetic field, the moving fluid experiences the Lorentz force that tends to suppress the flow velocities. This in turn reduces the heat transfer rate. Some authors have considered MHD flows in porous cavities. Examples are as follow:

Alchaar and his colleagues [1] studied free convection heat transfer of an electrically conducting fluid in a tilted porous cavity under the influence of a magnetic field. Bian et al. further extended this work to a shallow cavity [2] as well as an inclined one [3]. MHD free convection of a heat generation fluid in an inclined porous cavity was analyzed by Khanafer and Chamkha [4]. The generation of entropy during MHD free convection in a porous cavity was discussed by Mahmud and Fraser [5]. The combined effects of magnetic field orientation and heat generation on free convection in a porous cavity were noticed by Grosan et al. [6]. Pekmen and Tezer-Sezgin [7] simulated free convection in porous cavities with different cross-sections subjected to an oriented magnetic field. More recently, MHD free convection in a thermal and mass stratified porous cavity was investigated by Kumar et al. [8]. Additionally, MHD free convection in a porous cavity filled with $\mathrm{Fe}_{3} \mathrm{O}_{4}$-water nanofluid was pointed out by Geridönmez [9].

Recent years have witnessed an extensive study on heat transfer of nanofluids as a consequence of their appropriate thermophysical properties (eg., Mahfoud and Bendjaghloli [10]; Singh et al. [11]; Zahmatkesh and Naghedifar [12]; Zahmatkesh and Torshizi [13]; Zahmatkesh and Habibi [14]). Although a significant number of investigations on MHD free convection of nanofluids in cavities are available in the literature (e.g., Koopaee et al. [15,16], Akinshilo and Ilegbusi [17]), only a few recent studies have considered this problem in porous media.

MHD free convection and entropy generation of $\mathrm{Cu}$-water nanofluid in a porous cavity with time periodic heating on the mid portion of the left wall were discussed by Malik and Nayak [18]. Mansour et al. [19] studied the effect of viscous dissipation on entropy generation due to $\mathrm{MHD}$ free convection of $\mathrm{Cu}$-water nanofluid in a porous cavity with active parts. Sheikholeslami and Rokni [20] considered the problem of MHD free convection of CuOwater nanofluid in a porous complex-shaped cavity. Rashad et al. [21] extended the study of Grosan et al. [6] to

This paper was recommended for publication in revised form by Regional Editor Alibakhsh Kasaeian

${ }^{1}$ Department of Mechanical Engineering, Mashhad Branch, Islamic Azad University, Mashhad, Iran

*E-mail address: zahmatkesh5310@mshdiau.ac.ir

Orcid id: 0000-0003-2573-6789; 0000-0002-0579-8547

Manuscript Received 3 March 2018, Accepted 18 April 2018 
MHD free convection of a $\mathrm{Cu}$-water nanofluid in a porous cavity. In a recent attempt, they discussed entropy generation in MHD free convection of a nanofluid in an inclined porous cavity [22]. Effect of inclination angle of a porous square cavity on MHD free convection of several water-based nanofluids was investigated by Balla et al. [23]. More recently, Zahmatkesh and Shandiz [24] found optimum combination of nanoparticles, solid matrix, and base fluid for MHD heat transfer of nanofluids in porous cavities.

The aforesaid investigations are based on streamlines and isotherms. While streamlines can properly depict fluid flow, isotherms may not be suitable for heat transfer analysis since the heat flux lines are not perpendicular to isotherms in a convection-dominated problem. An accurate way to visualize heat transfer goes back to the use of heatlines [25]. The heatline visualization technique explains not only the heat trajectory but also the intensity of the heat flux inside the flow field of a convection heat transfer problem. So, free convection in a cavity can be best studied with the help of heatlines.

Saleh and Hashim [26] employed the heatline visualization technique to show heat transfer path in free convection within an inclined porous cavity with sinusoidal boundary conditions. They also undertook a numerical work to visualize conjugate heat transfer in square porous cavities [27]. A heatline visualization study for free convection of $\mathrm{Cu}$-water, $\mathrm{Al}_{2} \mathrm{O}_{3}$-water, and $\mathrm{TiO}_{2}$-water nanofluids in a porous cavity was presented by Zahmatkesh [28]. Alsabery et al. [29] used the heatline concept to analyze free convection in a trapezoidal cavity filled with a nanofluid porous layer and a non-Newtonian fluid layer. Heatlines and entropy generation during MHD free convection in a tilted sinusoidal corrugated porous cavity were discussed by Hussain [30]. Bondareva et al. [31] reported heatline patterns for MHD free convection of nanofluid in an inclined wavy open porous cavity with a local heater.

In light of the above discussion, the objective of the present study is to apply the heatline visualization technique to MHD free convection of nanofluid in a porous cavity. Attention is focused to examine how the orientation and magnitude of the imposed magnetic field may affect the flow strength and the resulting heat transfer for different Rayleigh numbers as well as the nanoparticles types and volume fractions. Most of the previous investigations have imposed a magnetic field in a direction parallel or perpendicular to the gravity vector while the case with an arbitrary orientation has not been fully explored. When the applied magnetic field is oblique, both the horizontal and vertical components of the Lorentz force will be present. This makes the consequences of the imposed magnetic field more complex.

\section{PROBLEM DEFINITION AND MATHEMATICAL FORMULATION}

MHD free convection of several water-based nanofluids in a square porous cavity subjected to an inclined magnetic field is considered in the present study. A schematic representation of this cavity is displayed in Figure 1.

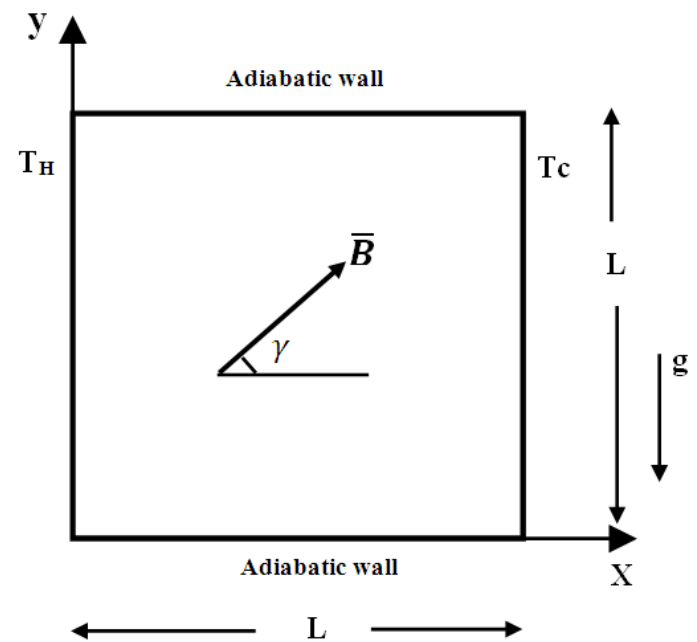

Figure 1. Schematic of the porous cavity subjected to an inclined magnetic field 
Journal of Thermal Engineering, Research Article, Vol. 6, No. 1, pp. 170-186, January, 2020

Table 1. Thermophysical properties of the nanoparticles and the base fluid in 300K [24].

\begin{tabular}{|c|c|c|c|c|c|}
\hline Material & $\sigma\left(\Omega^{-1} \mathrm{~m}^{-1}\right)$ & $\beta \times 10^{-5}\left(\mathrm{~K}^{-1}\right)$ & $k\left(W \mathrm{~m}^{-1} \mathrm{~K}^{-1}\right)$ & $\rho\left(\mathrm{kgm}^{-3}\right)$ & $C\left(\mathrm{~kg}^{-1} \mathrm{~K}^{-1}\right)$ \\
\hline Pure water & 0.05 & 21 & 0.613 & 997.1 & 4179 \\
\hline $\mathrm{Cu}$ & $59.6 \times 10^{6}$ & 1.67 & 401 & 8933 & 385 \\
\hline $\mathrm{Ag}$ & $6.3 \times 10^{6}$ & 1.89 & 429 & 10500 & 235 \\
\hline $\mathrm{Fe}_{3} \mathrm{O}_{4}$ & 25000 & 1.3 & 6 & 5200 & 670 \\
\hline
\end{tabular}

Here, the left wall is heated and the right wall is cooled. Meanwhile, the horizontal walls are thermally insulated. The direction of the gravity is downward while the orientation of the magnetic field forms an angle $\gamma$ with the horizontal axis. Under this circumstance, the fluid inside the cavity experiences both the buoyancy force resulting from heat transfer through the side walls and the Lorentz force due to the fluid motion within the magnetic field. The current study is based on the following assumptions:

1. The porous media is isotropic and homogenous.

2. The established flow is steady, incompressible, Newtonian, and laminar.

3. The Darcy model is adequate for the momentum equation.

4. Local thermal equilibrium (LTE) establishes between the nanofluid and the porous medium.

5. LTE exists between the nanoparticles and the base fluid.

6. The Boussinesq approximation is true to determine the variations of density in the body force term within the momentum equation, whereas, other thermophysical properties of the nanoparticles and the base fluid are constant with the numerical values presented in Table 1.

7. Viscous dissipation, the work done by the pressure change, radiation heat transfer, Joule heating effect, and induced magnetic field are negligible.

Within the framework of these assumptions, the continuity, momentum, and energy equations are:

$$
\begin{gathered}
\frac{\partial u}{\partial x}+\frac{\partial v}{\partial y}=0 \\
u=-\frac{K}{\mu_{n f}} \frac{\partial p}{\partial x}+\frac{\sigma_{n f} K B^{2}}{\mu_{n f}}\left(v \sin \gamma \cos \gamma-u \sin ^{2} \gamma\right) \\
v=-\frac{K}{\mu_{n f}} \frac{\partial p}{\partial y}+\frac{K g}{\mu_{n f}} \rho_{n f} \beta_{n f}\left(T-T_{C}\right)+\frac{\sigma_{n f} K B^{2}}{\mu_{n f}}\left(u \sin \gamma \cos \gamma-v \cos ^{2} \gamma\right) \\
u \frac{\partial T}{\partial x}+v \frac{\partial T}{\partial y}=\alpha_{n f}\left(\frac{\partial^{2} T}{\partial x^{2}}+\frac{\partial^{2} T}{\partial y^{2}}\right)
\end{gathered}
$$

Effective thermophysical properties of the nanofluid are determined as follows:

Effective heat capacitance:

$$
\rho_{n f} C_{n f}=(1-\phi) \rho_{f} C_{f}+\phi \rho_{p} C_{p}
$$

Effective electrical conductivity [32]:

$$
\frac{\sigma_{n f}}{\sigma_{f}}=1+\frac{3\left(\frac{\sigma_{p}}{\sigma_{f}}-1\right) \phi}{\left(\frac{\sigma_{p}}{\sigma_{f}}+2\right)-\left(\frac{\sigma_{p}}{\sigma_{f}}-1\right) \phi}
$$

Dynamic viscosity [33]: 


$$
\mu_{n f}=\frac{\mu_{f}}{(1-\phi)^{2.5}}
$$

Effective thermal conductivity [32]:

$$
\frac{k_{n f}}{k_{f}}=\frac{\left(k_{p}+2 k_{f}\right)-2 \phi\left(k_{f}-k_{p}\right)}{\left(k_{p}+2 k_{f}\right)+\phi\left(k_{f}-k_{p}\right)}
$$

Effective thermal diffusivity:

$$
\alpha_{n f}=\frac{k_{n f}}{\rho_{n f} C_{n f}}
$$

Effective thermal expansion coefficient [34]:

$$
\rho_{n f} \beta_{n f}=\phi \rho_{p} \beta_{P}+(1-\phi) \rho_{f} \beta_{p}-\phi(1-\phi)\left(\rho_{p}-\rho_{f}\right)\left(\beta_{p}-\beta_{f}\right)
$$

The last term in the bracket in the RHS of Equation 10 originates from the natural dependence of density on temperature. It has been neglected in most of the previous investigations that leads to a simple volume-averaging approximation for $\beta_{n f}$. Analysis of Zahmatkesh [35] demonstrates that such an approximation may introduce up to $30 \%$ error in $\overline{N u}$ predictions.

The momentum equations are cross-differentiated and then subtracted. So that the pressure terms are removed. After introducing stream function, the continuity equation will be satisfied and one arrives at the following governing equations:

$$
\frac{\partial^{2} \psi}{\partial x^{2}}+\frac{\partial^{2} \psi}{\partial y^{2}}=-\frac{K g}{\mu_{n f}} \rho_{n f} \beta_{n f} \frac{\partial T}{\partial x}-\frac{\sigma_{n f} K B^{2}}{\mu_{n f}}\left(\frac{\partial^{2} \psi}{\partial x^{2}} \cos ^{2} \gamma+\frac{\partial^{2} \psi}{\partial y^{2}} \sin ^{2} \gamma+2 \frac{\partial^{2} \psi}{\partial x \partial y} \sin \gamma \cos \gamma\right)
$$

and

$$
\frac{\partial \psi}{\partial y} \frac{\partial T}{\partial x}-\frac{\partial \psi}{\partial x} \frac{\partial T}{\partial y}=\alpha_{n f}\left(\frac{\partial^{2} T}{\partial x^{2}}+\frac{\partial^{2} T}{\partial y^{2}}\right)
$$

The following parameters are used to make the above equations dimensionless:

$$
\begin{gathered}
X=\frac{x}{L}, Y=\frac{y}{L}, \Psi=\frac{\psi}{\alpha_{f}}, \Theta=\frac{T-T_{C}}{T_{H}-T_{C}{ }^{2}} \\
R a=\frac{K g \rho_{f} \beta_{f}\left(T_{H}-T_{C}\right) L}{\mu_{f} \alpha_{f}}, H a^{2}=\frac{\sigma_{f} B_{0}{ }^{2} K}{\mu_{f}}
\end{gathered}
$$

Here, $R a$ is the Rayleigh number and $H a$ is the Hartmann number.

Substituting the dimensionless parameters into the governing equations yields: 


$$
\begin{aligned}
\frac{\partial^{2} \Psi}{\partial X^{2}}+\frac{\partial^{2} \Psi}{\partial Y^{2}}=-R a(1-\phi)^{2.5}\left[(1-\phi)+\phi\left(\frac{\rho_{p}}{\rho_{f}}\right)\left(\frac{\beta_{p}}{\beta_{f}}\right)-\phi(1-\phi)\left(\frac{\rho_{p}}{\rho_{f}}-1\right)\left(\frac{\beta_{p}}{\beta_{f}}-1\right)\right] \frac{\partial \theta}{\partial X} \\
-H a^{2}(1-\phi)^{2.5}\left[\frac{\partial^{2} \Psi}{\partial X^{2}} \cos ^{2} \gamma+2 \frac{\partial^{2} \Psi}{\partial X \partial Y} \sin \gamma \cos \gamma+\frac{\partial^{2} \Psi}{\partial Y^{2}} \sin ^{2} \gamma\right][1 \\
\left.+\frac{3\left(\frac{\sigma_{p}}{\sigma_{f}}-1\right) \phi}{\left(\frac{\sigma_{p}}{\sigma_{f}}+2\right)-\left(\frac{\sigma_{p}}{\sigma_{f}}-1\right) \phi}\right]
\end{aligned}
$$

and

$$
\frac{\partial \Psi}{\partial Y} \frac{\partial \Theta}{\partial X}-\frac{\partial \Psi}{\partial X} \frac{\partial \Theta}{\partial Y}=\frac{1}{1-\phi+\phi \frac{\rho_{p} C_{p}}{\rho_{f} C_{f}}}\left[\frac{\partial^{2} \Theta}{\partial X^{2}}+\frac{\partial^{2} \Theta}{\partial Y^{2}}\right]\left[\frac{k_{p}+2 k_{f}-2 \phi\left(k_{f}-k_{p}\right)}{k_{p}+2 k_{f}+\phi\left(k_{f}-k_{p}\right)}\right]
$$

The associated boundary conditions are:

$$
\begin{array}{lc}
X=0,0<Y<1: & \Psi=0, \Theta=1 \\
X=1,0<Y<1: & \Psi=0, \Theta=0 \\
0<X<1, Y=0: & \Psi=0, \partial \Theta / \partial Y=0 \\
0<X<1, Y=1: & \Psi=0, \partial \Theta / \partial Y=0
\end{array}
$$

The heat function, $h$, applied to a nanofluid problem is governed by [28]:

$$
\begin{gathered}
-\frac{\partial h}{\partial x}=\rho_{n f} C_{n f} v\left(T-T_{C}\right)-k_{n f} \frac{\partial T}{\partial y} \\
\frac{\partial h}{\partial y}=\rho_{n f} C_{n f} u\left(T-T_{C}\right)-k_{n f} \frac{\partial T}{\partial x}
\end{gathered}
$$

If one introduces the dimensionless heat function as:

$$
\Pi=\frac{h}{k_{f}\left(T_{H}-T_{C}\right)}
$$

these relations are converted to the following dimensionless form:

$$
\begin{aligned}
& \frac{\partial \Pi}{\partial X}=\frac{\rho_{n f} C_{n f}}{\rho_{f} c_{f}} \theta \frac{\partial \Psi}{\partial X}+\frac{k_{n f}}{k_{f}} \frac{\partial \theta}{\partial Y} \\
& \frac{\partial \Pi}{\partial Y}=\frac{\rho_{n f} C_{n f}}{\rho_{f} c_{f}} \theta \frac{\partial \Psi}{\partial Y}-\frac{k_{n f}}{k_{f}} \frac{\partial \theta}{\partial X}
\end{aligned}
$$

Table 2. Comparison of the average Nusselt number for a $\mathrm{Cu}$-water nanofluid in a triangular cavity 
Journal of Thermal Engineering, Research Article, Vol. 6, No. 1, pp. 170-186, January, 2020

with those of previous works

\begin{tabular}{|c|c|c|c|c|}
\hline & \multicolumn{2}{|c|}{$R a=500$} & \multicolumn{2}{c|}{$R a=1000$} \\
\cline { 2 - 5 } & $\phi=0$ & $\phi=0.2$ & $\phi=0$ & $\phi=0.2$ \\
\hline Sun and Pop [36] & 9.66 & 9.42 & 13.9 & 12.85 \\
\hline Chamkha and Ismael [37] & 9.52 & 9.44 & 13.60 & 12.82 \\
\hline Current work & 9.53 & 9.41 & 13.67 & 12.81 \\
\hline
\end{tabular}

We now assume that $h$ is a continuous function to its second-order derivatives. So, the above equations lead to a partial differential equation as:

$$
\frac{\partial^{2} \Pi}{\partial X^{2}}+\frac{\partial^{2} \Pi}{\partial Y^{2}}=\left[1-\phi+\phi \frac{\rho_{p} C_{p}}{\rho_{f} C_{f}}\right]\left[\frac{\partial}{\partial X}\left(\Theta \frac{\partial \Psi}{\partial X}\right)+\frac{\partial}{\partial Y}\left(\Theta \frac{\partial \Psi}{\partial Y}\right)\right]
$$

Solution of this equation yields the $\Pi$ values for the inside nodes. So, drawing of the iso-lines of the heat function sketches the heatlines.

If we take the heat function value at the origin point to be zero, the boundary conditions for Equation 22 become [28]:

$$
\begin{array}{lc}
0<X<1, Y=0: & \Pi(X, 0)=\Pi(0,0)=0 \\
0<X<1, Y=1: & \Pi(X, 1)=\Pi(0,1) \\
X=0,0<Y<1: & \Pi(0, Y)=\Pi(0,0)+\int_{0}^{1} N u d Y \\
X=1,0<Y<1: & \Pi(1, Y)=\Pi(1,0)+\int_{0}^{1} N u d Y
\end{array}
$$

\section{COMPUTATIONAL METHOD}

The computations are performed adopting the finite-volume method. The system of dimensionless governing equations (Equation 14, 15 and 22) is integrated over the control volumes that leads to a system of algebraic equations. The resulting equations in conjunction with the aforesaid boundary conditions are thereafter solved by a line-by-line iterative method. The method sweeps the domain of integration along the $\mathrm{x}$ and $\mathrm{y}$ axes and uses Tri-Diagonal Matrix Algorithm (TDMA) to solve the system of equations. The numerical method was implemented in a FORTRAN software.

The following convergence criterion is employed throughout the computations:

$$
\left|\varepsilon_{i, j}^{n+1}-\varepsilon_{i, j}^{n}\right|<10^{-5}
$$

Here, $\varepsilon$ denotes for $\Psi, \theta$, or $\Pi$. $n$ is the time level while $i$ and $j$ are the cell index.

As the successive iterations lead to a converged solution, the local and the average Nusselt numbers at the left wall are obtained as:

$$
N u=\frac{h L}{k_{f}}=-\frac{k_{n f}}{k_{f}}\left[\frac{\partial \Theta}{\partial X}\right]_{X=0}
$$




$$
\overline{N u}=\int_{0}^{1} N u d Y
$$

In order to achieve a suitable grid for our calculations, a grid independence study is performed. We observed that refinement of the grid from $200 \times 200$ to $300 \times 300$ may not change the $\overline{N u}$ values more than $1 \%$. So, a $200 \times 200$ grid is chosen for further computations.

Prior to presenting the results associated with the objectives of this study, the FORTRAN code is validated. Two different tests are considered to this aim. In the first test, in the absence of external magnetic field, free convection of $\mathrm{Cu}$-water nanofluid in a triangular cavity is studied, as reported in Table 2. In the second, free convection of pure fluid $(\phi=0)$ in a square porous cavity with differentially-heated vertical walls and insulated horizontal walls in the presence of a downward magnetic field is concerned, as depicted in Figure 2. Notice that the results of the current model bear a strong resemblance with those of the previous works. These favorable comparisons lend confidence in the numerical results to be reported subsequently.

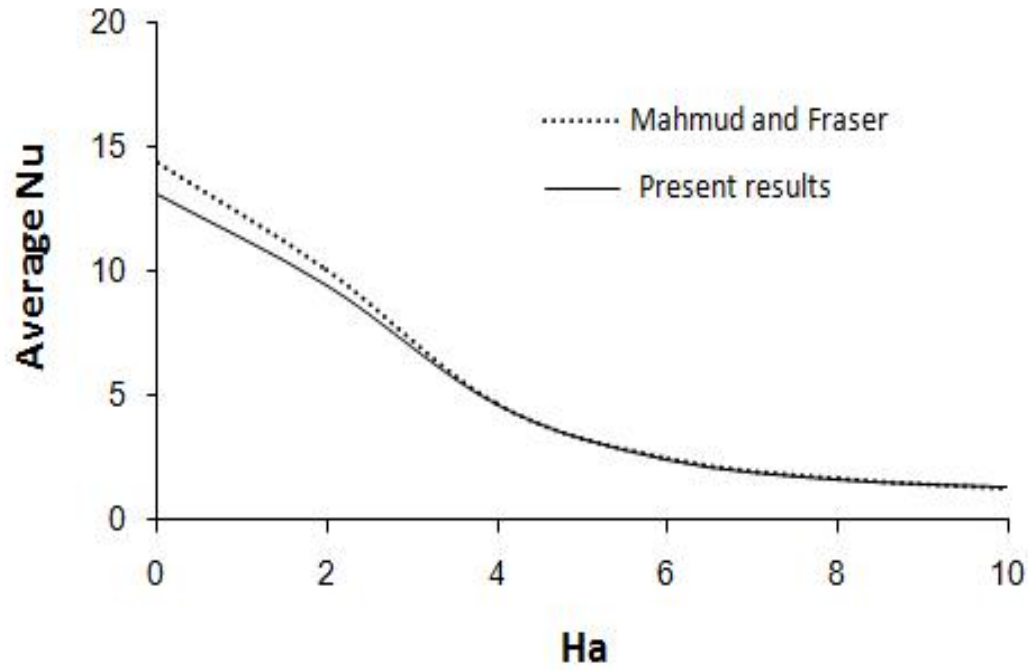

Figure 2. Comparison of the current values of the average Nusselt number with those of Mahmud and Fraser [5]

$$
(R a=1000, \gamma=-\pi / 2, \phi=0)
$$

\section{SIMULATION RESULTS}

In what concerns the distributions of streamlines (left), isotherms (middle), and heatlines (right), simulation results for an Ag-water nanofluid with $H a=1, \gamma=0$, and $\phi=4 \%$ under different Rayleigh numbers are plotted in Figure 3. Here, results in the absence of magnetic field are also depicted by dashed lines for comparison. Additionally, values of $\left|\Psi_{\max }\right|$ and $\overline{N u}$ for each case are provided with $\left|\Psi_{\max }\right|$ being a measure of the convection vigor.

Notice in the streamlines and the isotherms that the fluid becomes heated and rises up near the left wall; it changes its direction when reaching the top wall, then, it loses heat at the right wall and moves downward. As a result, one may find a unicellular flow circulating within the cavity.

Heat flow template inside the cavity is explained by the heatlines that depict the trajectory and magnitude of the heat energy. Notice that the heatlines start from the left wall and stop at the right wall. This indicates that heat is absorbed from the left surface and released to the right surface.

Inspection of the results at different Rayleigh numbers in Figure 3 demonstrates that when $R a=10$, the established flow is very weak, the isotherms are almost parallel to the vertical walls, and the heat function values are very low. Moreover, the heatlines are approximately perpendicular to the isotherms. This is not surprising since thermal conduction is the dominant heat transfer mechanism there. Notice, however, that increase in $R a$ intensifies the fluid motion and deforms the isotherms. This results in heat transfer enhancement as indicated by the increased 
$\overline{N u}$ and $\left|\pi_{\max }\right|$ values. Moreover, expansion appears in the passive heat transfer zone within the cavity with negative heat function values wherein heat is rotated without affecting the walls heat exchange.

\begin{tabular}{|c|}
\hline $\begin{array}{l}\mathbf{R a}=\mathbf{1 0} \\
\left|\Psi_{\max }\right|=0.578 \\
\mathrm{Nu}=1.163 \\
\left|\pi_{\max }\right|=1.161\end{array}$ \\
\hline $\begin{array}{l}\text { Dashed lines: } \\
\left|\Psi_{\max }\right|=0.865 \\
N u=1.215 \\
\left|\pi_{\max }\right|=1.213\end{array}$ \\
\hline $\begin{array}{l}\mathbf{R a}=\mathbf{1 0 0} \\
\left|\Psi_{\max }\right|=4.176 \\
N u=2.654 \\
\left|\pi_{\max }\right|=2.842\end{array}$ \\
\hline $\begin{array}{l}\text { Dashded lines: } \\
\left|\Psi_{\max }\right|=5.597 \\
N u=3.566 \\
\left|\pi_{\max }\right|=3.562\end{array}$ \\
\hline $\begin{array}{l}\mathbf{R a}=\mathbf{1 0 0 0} \\
\left|\Psi_{\max }\right|=17.524 \\
\overline{N u}=10.785 \\
\left|\pi_{\max }\right|=13.641\end{array}$ \\
\hline $\begin{array}{l}\text { Dashed lines: } \\
\left|\Psi_{\max }\right|=24.050 \\
\overline{N u}=15.254 \\
\left|\pi_{\max }\right|=15.230\end{array}$ \\
\hline
\end{tabular}

$\boldsymbol{R a}=\mathbf{1 0}$
$\left|\Psi_{\max }\right|=0.578$
$\overline{N u}=1.163$
$\left|\pi_{\max }\right|=1.161$

Dashed lines:

$\left|\Psi_{\max }\right|=0.865$

$\overline{N u}=1.215$

$\pi_{\max } \mid=1.213$

$\mathbf{R a}=\mathbf{1 0 0}$

$\overline{N u}=2.654$

$\left|\pi_{\max }\right|=2.842$

Dashded lines:

$\left|\Psi_{\max }\right|=5.597$

$\overline{N u}=3.566$

$\left|\pi_{\max }\right|=3.562$

$\mathbf{R a}=\mathbf{1 0 0 0}$

$\left|\Psi_{\max }\right|=17.52$

$\overline{N u}=10.785$

Dashed lines:

$\overline{N u}=15.254$

$\left|\pi_{\max }\right|=15.230$ $\theta$
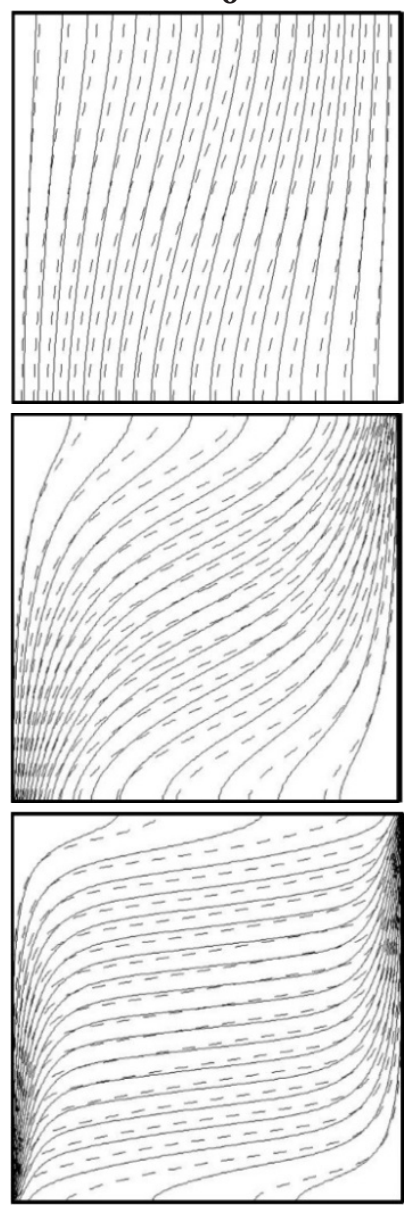
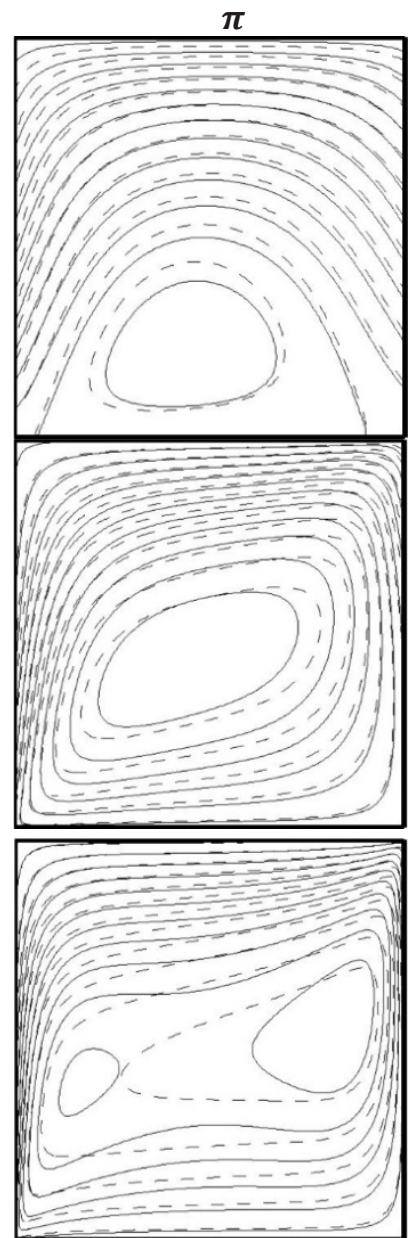

Figure 3. Distributions of streamlines, isotherms, and heatlines for an Ag-water nanofluid ( $H a=1, \gamma=0, \phi=4 \%$ ); dashed lines correspond to $\mathrm{Ha}=0$

Notice also that the heatlines are no longer perpendicular to the isotherms and pattern of the heatlines takes the shape of the streamlines. These observations are attributed to the domination of the convection heat transfer. The presence of significant convection is also exhibited in the enhanced value of $\left|\Psi_{\text {max }}\right|$. The dense heatlines that appear near the middle portion of the side walls at $R a=1000$ indicate higher heat transfer rates there.

Comparing the contour maps in the solid lines with those in the dashed lines in Figure 3 shows that the imposition of the magnetic field is not so influential at $R a=10$. Notice, however, that with rise in the Rayleigh number, the role of the imposed magnetic field increases since discrepancies that appear between the results in the presence of the magnetic field and those without it become pronounced.

Scrutiny of the numerical values of $\left|\Psi_{\max }\right|, \overline{N u}$, and $\left|\pi_{\max }\right|$ for the current cases demonstrates that with the imposition of the magnetic field, the flow strength becomes weakened and the resulting heat transfer is suppressed. Notice that the retarding effect is more remarkable at higher Rayleigh numbers. This behavior is expected since the Lorentz force is directly proportional to the flow velocity. Heat transfer deteriorations as a result of the magnetic field imposition are about 4, 26, and 29 percents for the Rayleigh numbers of 10, 100, and 1000, respectively. 
Results presented in Figure 3 correspond to a horizontal magnetic field. To analyze how orientation of the imposed magnetic field may be influential, simulation results for oblique $(\gamma=\pi / 4)$ and vertical magnetic fields are presented in Figure 4 and Figure 5, respectively.

\begin{tabular}{|c|}
\hline $\begin{array}{l}\mathbf{R a}=\mathbf{1 0} \\
\left|\Psi_{\max }\right|=0.572 \\
\overline{N u}=1.162 \\
\left|\pi_{\max }\right|=1.159\end{array}$ \\
\hline $\begin{array}{l}\text { Dashed lines: } \\
\left|\Psi_{\max }\right|=0.865 \\
\overline{N u}=1.215 \\
\left|\pi_{\max }\right|=1.213\end{array}$ \\
\hline $\begin{array}{l}\mathbf{R a}=\mathbf{1 0 0} \\
\left|\Psi_{\max }\right|=4.131 \\
\overline{N u}=2.695 \\
\left|\pi_{\max }\right|=2.831\end{array}$ \\
\hline $\begin{array}{l}\text { Dashded lines: } \\
\left|\Psi_{\max }\right|=5.597 \\
\overline{N u}=3.566 \\
\left|\pi_{\max }\right|=3.562\end{array}$ \\
\hline $\begin{array}{l}\boldsymbol{R a}=\mathbf{1 0 0 0} \\
\left|\Psi_{\max }\right|=18.844 \\
\overline{N u}=11.960 \\
\left|\pi_{\max }\right|=13.473\end{array}$ \\
\hline $\begin{array}{l}\text { Dashed lines: } \\
\left|\Psi_{\max }\right|=24.050 \\
\overline{N u}=15.254 \\
\left|\pi_{\max }\right|=15.230\end{array}$ \\
\hline
\end{tabular}
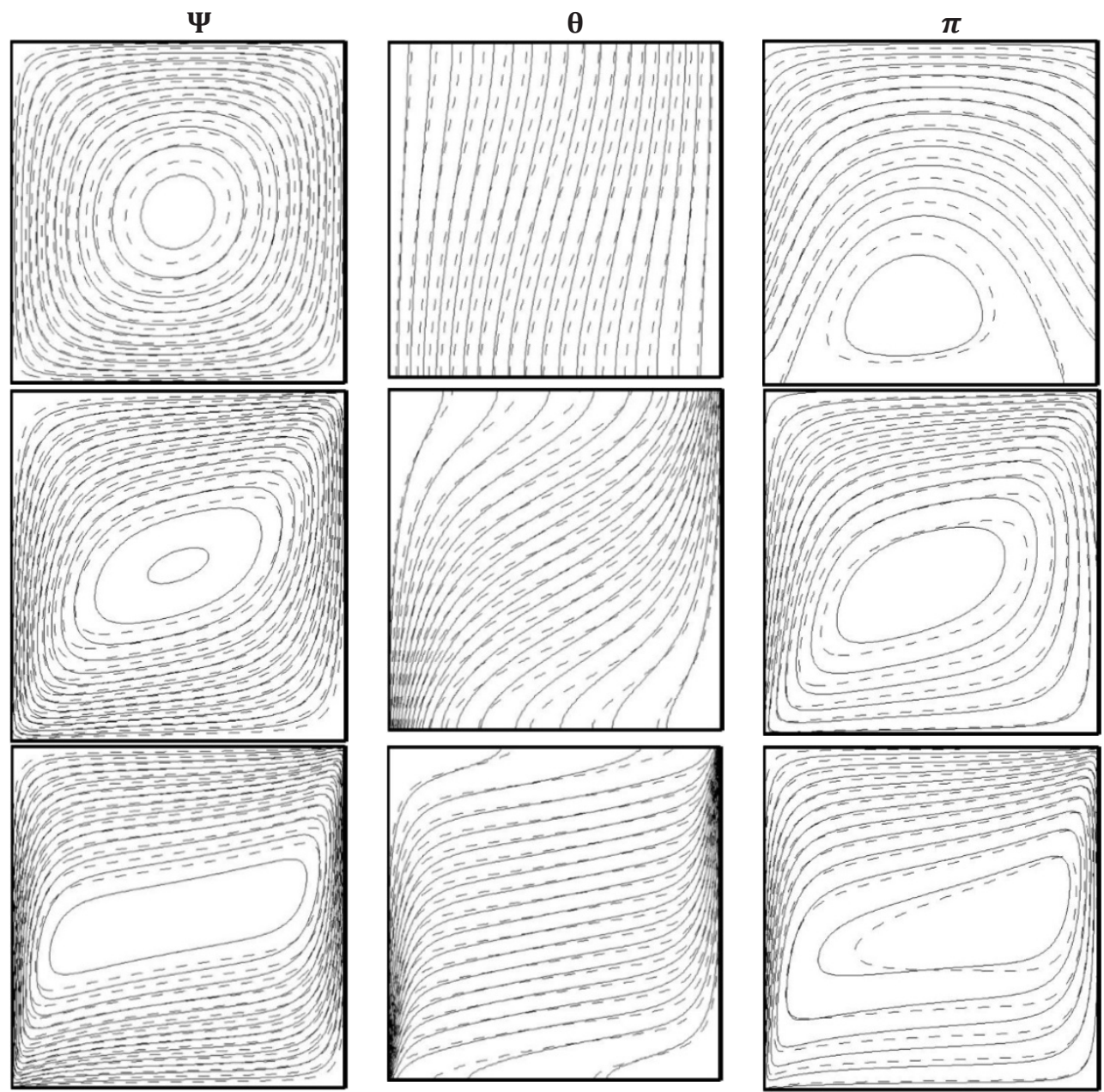

Figure 4. Distributions of streamlines, isotherms, and heatlines for an Ag-water nanofluid ( $\mathrm{Ha}=1, \gamma=\pi / 4, \phi=4 \%)$; dashed lines correspond to $\mathrm{Ha}=0$

Comparing the streamlines, isotherms, and heatlines of Figure 4 and Figure 5 with those of Figure 3 demonstrates that suppression effect of the horizontal magnetic field is more remarkable than the vertical or inclined ones. Specifically, for an Ag-water nanofluid with $\mathrm{Ha}=1$ and $\phi=4 \%$, when the magnetic field inclination angle changes form vertical to horizontal, the flow strength diminishes up to about $17 \%$ and the resulting heat transfer deteriorates up to about $23 \%$. This occurs since temperature gradients and the buoyancy force are strong near the side walls. So, the vertical component of velocity is much higher than the horizontal one, as indicated in the dense streamlines near the right/left walls. This leads the Lorentz force to be greater in a horizontal magnetic field. The observed trend is in accord with previous findings of Grosan et al. [6] and Rashad et al. [21].

The overall effects of the orientation and magnitude of the imposed magnetic field on the heat transfer rate in a range of the Rayleigh number are demonstrated in Figure 6. The presented results correspond to an Ag-water nanofluid with $\phi=4 \%$. In a general way notice that increment in the magnetic field strength deteriorates the heat transfer rate. This effect, however, diminishes with rise in the magnetic field inclination angle. It is also obvious that with increase in the Rayleigh number, the role of the imposed magnetic field on the suppression of the heat transfer 
enhances. These behaviors are expected and were discussed previously. Notice that as the Hartmann number passes from 0 to 1 at $R a=1000$, the heat transfer deteriorates to about 29, 22, and 8 percents for $\gamma=0, \pi / 4$, and $\pi / 2$, respectively.

$\Psi$

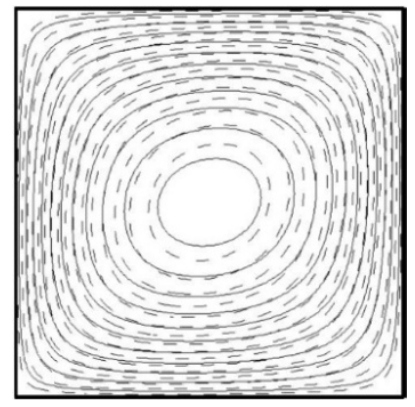

$R a=100$

$\left|\Psi_{\max }\right|=4.164$

$\overline{N u}=2.829$

$\left|\pi_{\max }\right|=2.926$

Dashed lines:

$\left|\Psi_{\max }\right|=5.597$

$N u=3.566$

$\left|\pi_{\max }\right|=3.562$

$R a=1000$

$\left|\Psi_{\max }\right|=21.230$

$\overline{N u}=13.989$

$\left|\pi_{\max }\right|=15.241$

Dashed lines:

$\left|\Psi_{\max }\right|=24.050$

$\overline{N u}=15.254$

$\left|\pi_{\max }\right|=15.230$
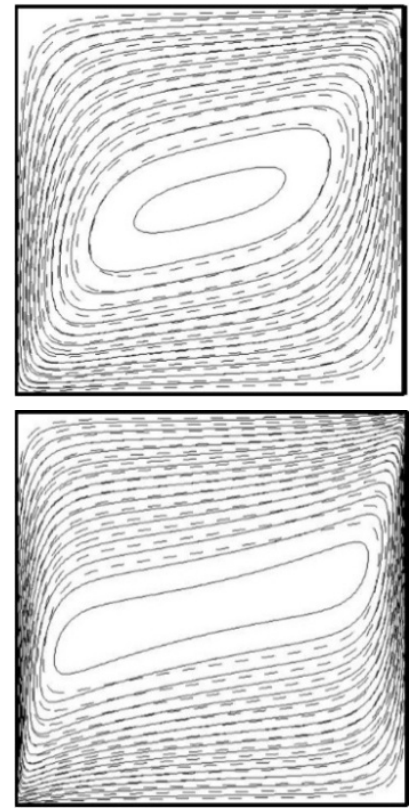

$\theta$
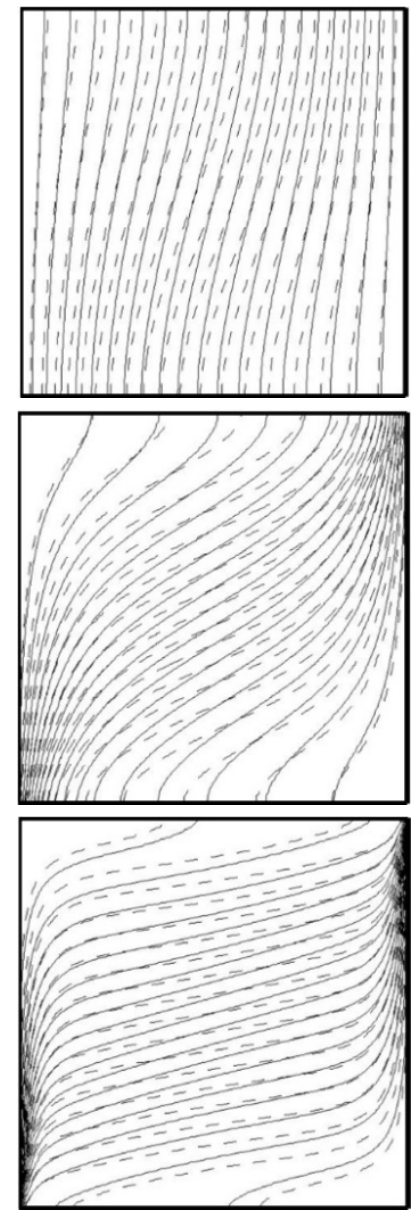

$\pi$

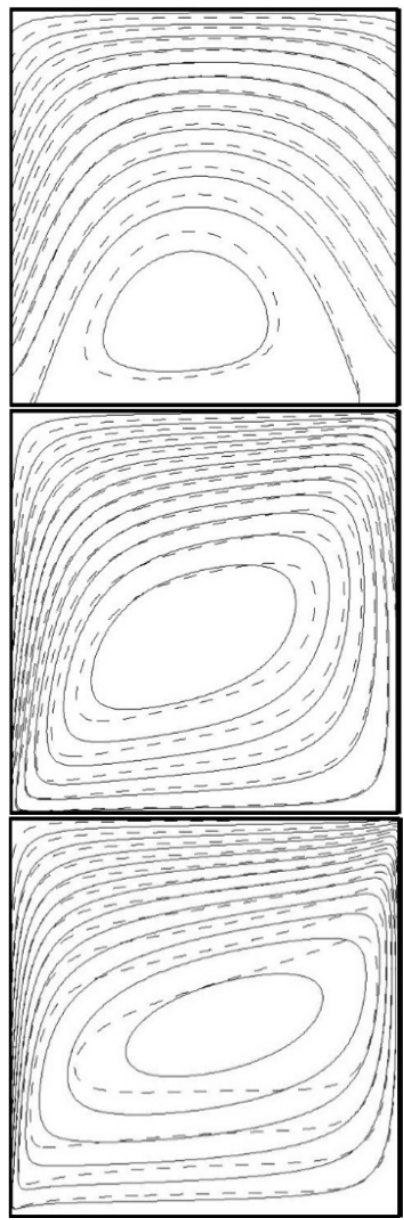

Figure 5. Distributions of streamlines, isotherms, and heatlines for an Ag-water nanofluid ( $H a=1, \gamma=\pi / 2, \phi=4 \%)$; dashed lines correspond to $H a=0$

Now, the consequences of the nanoparticles fraction on the establishment of the flow and thermal fields as well as the heat transfer characteristics are discussed. To this aim, the streamlines, isotherms, and heatlines at different Rayleigh numbers for $\phi=4 \%$ and $8 \%$ are plotted in Figure 7 and Figure 8, respectively. The results owe to $H a=1$ and $\gamma=0$ with the dashed lines belonging to the base fluid (i.e., pure water). Inspection of the results demonstrates that the presence of the nanoparticles is more influential at higher Rayleigh numbers. Notice that with a $4 \%$ particles addition, the strength of the induced flow improves up to about 19 percent. One may also find up to about 15 percent elevation in $\overline{N u}$ with this particles addition. The corresponding values for a $8 \%$ particles addition are 32 and 27 percents, respectively. The intensified buoyancy-driven flow and the enhanced heat transfer are in agreement with the observations of Bourantas et al. [34] but in conflict with the evidences of Rashad et al. [21]. 


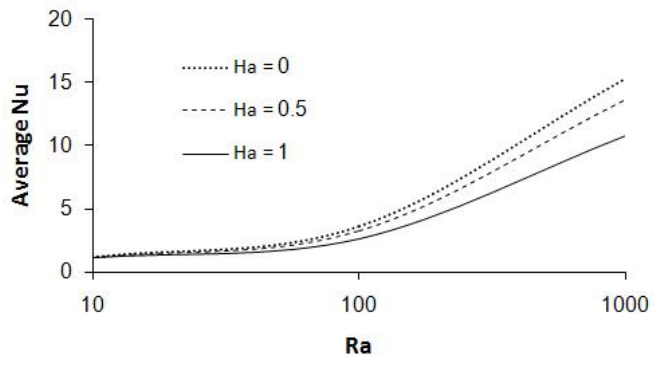

(a) $\gamma=0$

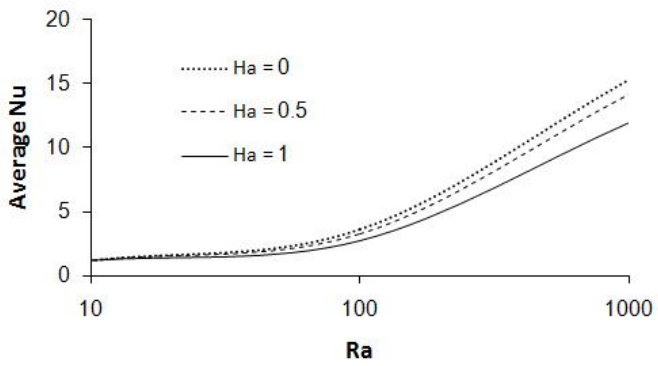

(b) $\gamma=\pi / 4$

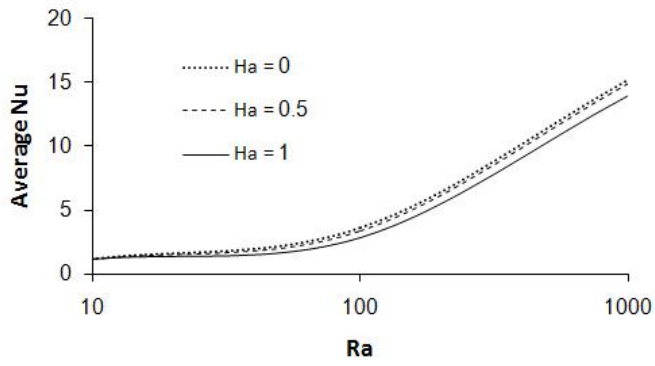

(c) $\gamma=\pi / 2$

Figure 6. The average Nusselt number vs. the Rayleigh number for different orientations and magnitudes of the magnetic field (Ag-water nanofluid with $\phi=4 \%$ )

$$
\begin{aligned}
& \boldsymbol{R a}=\mathbf{1 0} \\
& \left|\Psi_{\max }\right|=0.578 \\
& \overline{N u}=1.164 \\
& \left|\pi_{\max }\right|=1.161
\end{aligned}
$$
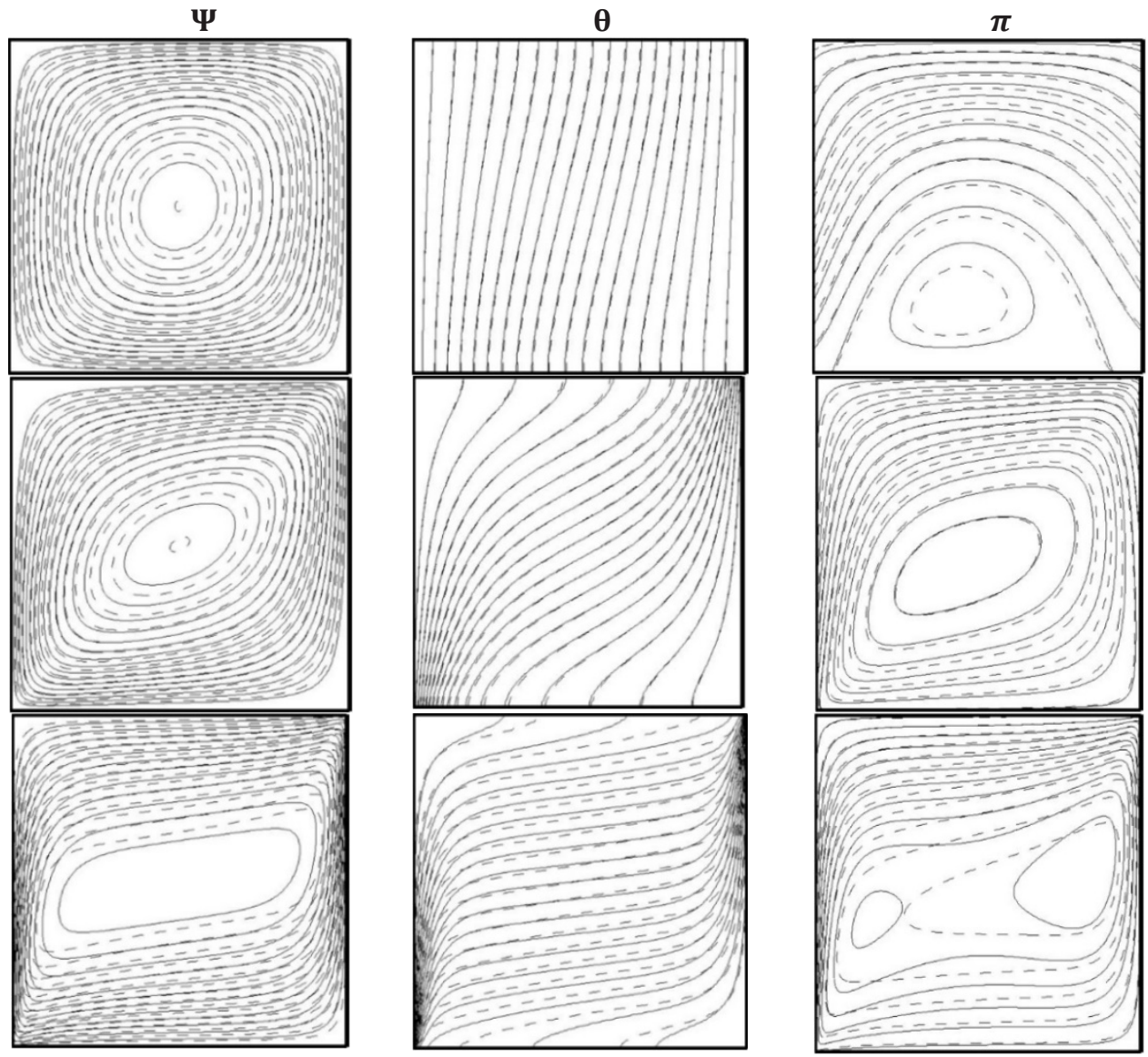

Figure 7. Distributions of streamlines, isotherms, and heatlines for an $\mathrm{Ag}$-water nanofluid ( $H a=1, \gamma=0, \phi=4 \%)$; dashed lines correspond to pure water 
The contradiction is attributed to the method they employed for the determination of the effective thermal expansion coefficient. Indeed, the study of Rashad and his coworkers relays on a simple volume-averaging approximation for $\rho_{n f} \beta_{n f}$ while Bourantas et al. employed a more elaborate model to this aim, i.e., Equation 10. To demonstrate this effect, results of the two approaches in terms of flow intensity and the average Nusselt number at different nanoparticles fractions and Rayleigh numbers are provided in Table 3. The presented results correspond to an Ag-water nanofluid in the absence of magnetic field. In a general way notice that with the Bourantas correlation, both inclusion of the nanoparticles and increasing their volume fraction lead to enhancement in $\left|\Psi_{\max }\right|$ and $\overline{N u}$, that is in accord with Bourantas et al. [34]. In contrast to this and similar to Rashad et al. [21] notice that the flow intensity and the average Nusselt number are decreasing functions of the nanoparticles fractions during the volumeaveraging approximation. The direct relation of $\overline{N u}$ with $\phi$ that appears in $R a=10$ goes back to the enhanced thermal conductivity in this conduction dominant regime.

$$
\begin{aligned}
& R a=10 \\
& \left|\Psi_{\max }\right|=0.637 \\
& \overline{N u}=1.300 \\
& \left|\pi_{\max }\right|=1.297 \\
& \text { Dashed lines: } \\
& \left|\Psi_{\text {max }}\right|=0.484 \\
& \overline{N u}=1.032 \\
& \left|\pi_{\max }\right|=1.029 \\
& \boldsymbol{R a}=\mathbf{1 0 0} \\
& \left|\Psi_{\text {max }}\right|=4.643 \\
& \overline{N u}=2.912 \\
& \left|\pi_{\max }\right|=3.170 \\
& \text { Dashed lines: } \\
& \left|\Psi_{\max }\right|=3.538 \\
& \overline{N u}=2.297 \\
& \left|\pi_{\max }\right|=2.389 \\
& \boldsymbol{R a}=\mathbf{1 0 0 0} \\
& \left|\Psi_{\text {max }}\right|=19.580 \\
& \overline{N u}=11.843 \\
& \left|\pi_{\max }\right|=15.247 \\
& \left|\Psi_{\max }\right|=14.962 \\
& \overline{N u}=9.357 \\
& \left|\pi_{\max }\right|=11.516
\end{aligned}
$$
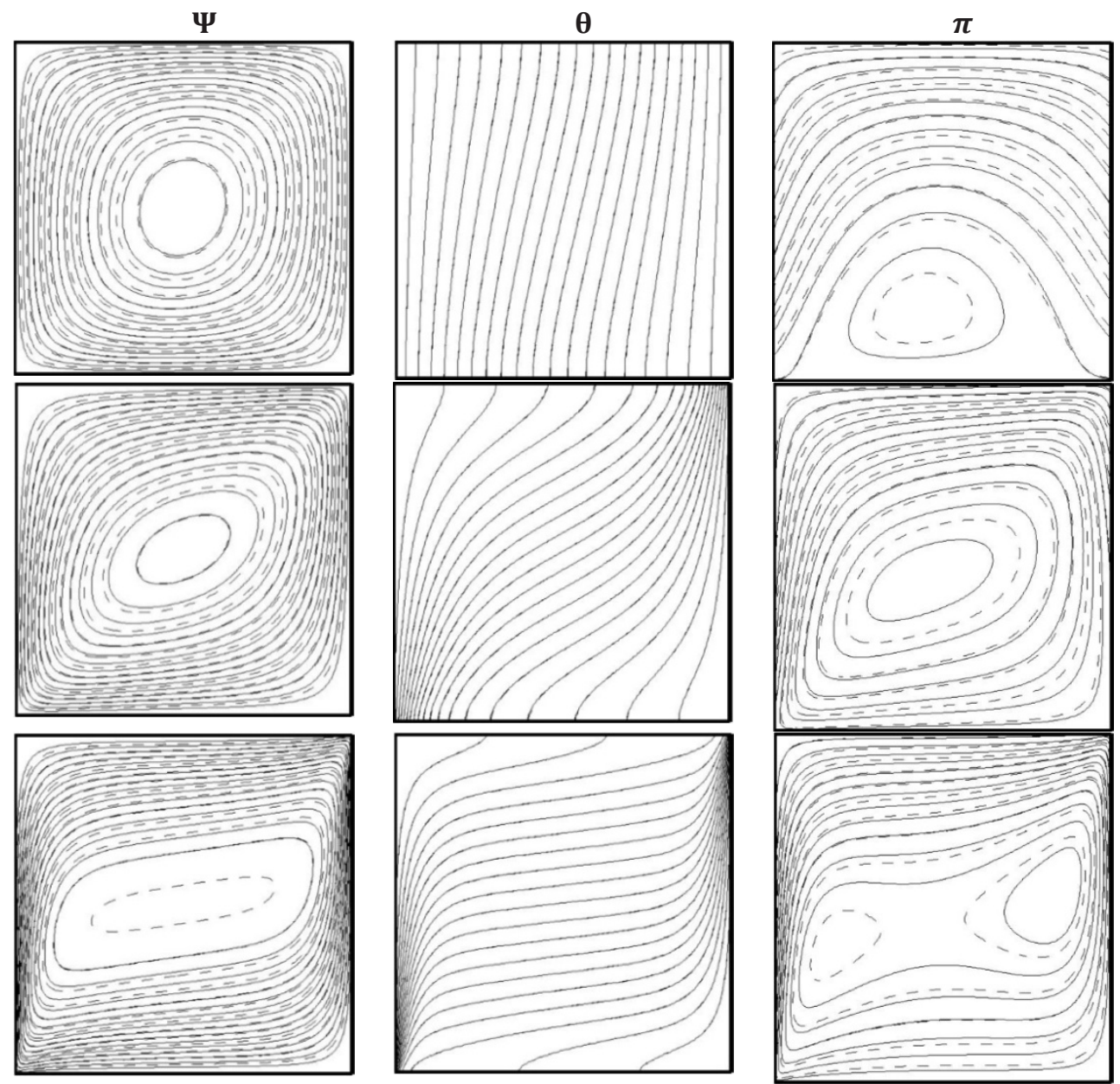

Figure 8. Distributions of streamlines, isotherms, and heatlines for an Ag-water nanofluid ( $\mathrm{Ha}=1, \gamma=0, \phi=8 \%)$; dashed lines correspond to pure water

Figure 9 is constructed to show the effect of the nanoparticles fraction on the average Nusselt number for a range of the Rayleigh number. The presented results correspond to $\gamma=0, \pi / 4$, and $\pi / 2$ with $H a=1$. Here, results in the absence of the magnetic field are also provided. Notice that in all of the current cases, heat transfer improves as a result of increase in the nanoparticles fraction. Numerical results show that, in the current range of $R a$, the $\overline{N u}$ augmentation due to increase in $\phi$ from 0 to 8 percent is about 27 percent both in the presence and the absence of the magnetic field. 
Journal of Thermal Engineering, Research Article, Vol. 6, No. 1, pp. 170-186, January, 2020

Table 3. Comparison of the results of the volume-averaging approximation and those of the Bourantas correlation in different conditions (Ag-water nanofluid without magnetic field)

\begin{tabular}{|c|c|c|c|c|c|}
\hline \multirow{3}{*}{$R$} & \multirow{2}{*}{$\phi$} & \multicolumn{2}{|c|}{ Volume-averaging } & \multicolumn{2}{c|}{ Bourantas correlation } \\
\cline { 2 - 5 } & & $\left|\Psi_{\max }\right|$ & $\overline{N u}$ & $\left|\Psi_{\max }\right|$ & $\overline{N u}$ \\
\hline \multirow{3}{*}{$R a=10$} & 0 & 0.722 & 1.073 & 0.722 & 1.073 \\
\cline { 2 - 6 } & 0.04 & 0.656 & 1.175 & 0.865 & 1.215 \\
\cline { 2 - 6 } & 0.08 & 0.592 & 1.293 & 0.956 & 1.355 \\
\hline \multirow{3}{*}{$R a=100$} & 0 & 4.728 & 3.066 & 4.728 & 3.066 \\
\cline { 2 - 6 } & 0.04 & 4.568 & 2.945 & 5.597 & 3.566 \\
\cline { 2 - 6 } & 0.08 & 4.377 & 2.827 & 6.237 & 3.911 \\
\cline { 2 - 6 } & 0 & 20.444 & 13.180 & 20.444 & 13.180 \\
\cline { 2 - 6 } & 0.04 & 20.358 & 12.904 & 24.050 & 15.254 \\
\hline
\end{tabular}

It is interesting to investigate the effect of the nanoparticles type on the current flow problem. To this aim, simulation results of an Ag-water nanofluid at $R a=1000, H a=1, \gamma=0$, and $\phi=4 \%$ are compared with those of a $\mathrm{Cu}$-water and a $\mathrm{Fe}_{3} \mathrm{O}_{4}$-water ones in Figure 10. Here, results of the pure water are also depicted by the dashed lines. Evidently, the Ag-water nanofluid has the utmost heat transfer among the other ones that is attributed to the highest thermal conductivity of the silver nanoparticles.

Finally, Figure 11 discusses the influence of the nanoparticles type on the average Nusselt number for a range of the Rayleigh number with $\phi=4 \%$. Notice that heat transfer performance of the Ag-water nanofluid is always higher than the $\mathrm{Cu}$-water and $\mathrm{Fe}_{3} \mathrm{O}_{4}$-water ones, both in the absence and the presence of the magnetic field. Maximum improvements in $\overline{N u}$ that appear as a result of the addition of the $\mathrm{Ag}, \mathrm{Cu}$, and $\mathrm{Fe}_{3} \mathrm{O}_{4}$ nanoparticles to pure water are about 16, 13, and 9 percents, respectively.

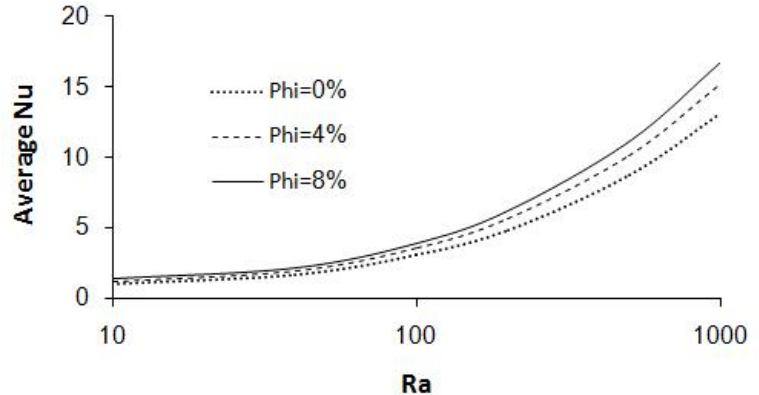

(a) $\mathrm{Ha}=0$

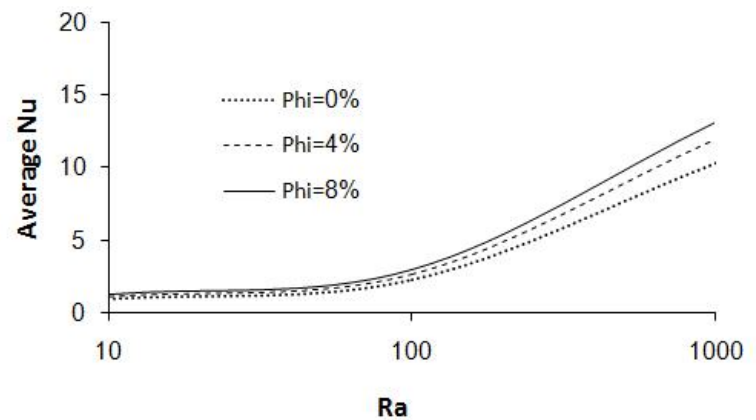

(c) $H a=1, \gamma=\pi / 4$

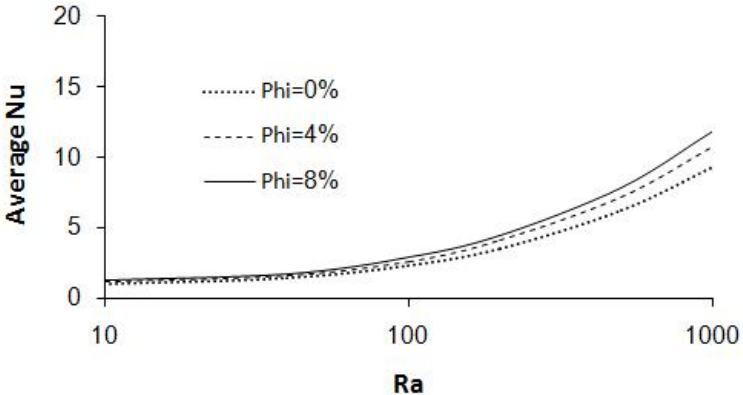

(b) $H a=1, \gamma=0$

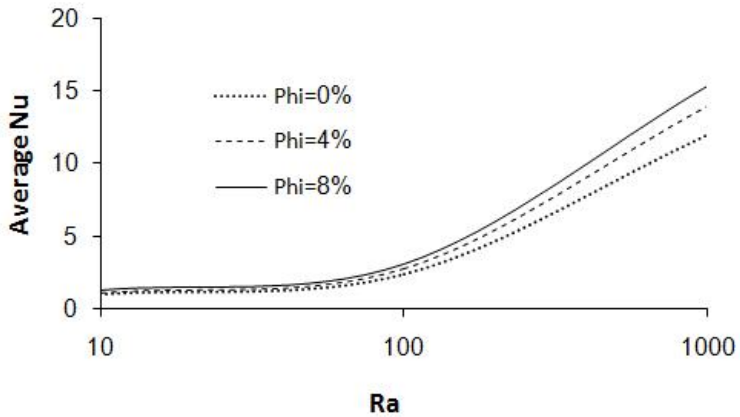

(d) $H a=1, \gamma=\pi / 2$

Figure 9. The average Nusselt number vs. the Rayleigh number for different nanoparticles fractions of an Ag-water nanofluid 


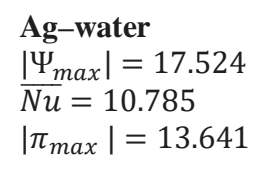

Cu-water

$\left|\Psi_{\max }\right|=17.009$

$\overline{N u}=10.555$

$\left|\pi_{\max }\right|=13.180$

$\mathrm{Fe}_{3} \mathrm{O}_{4}$-water

$\left|\Psi_{\max }\right|=15.678$

$\overline{N u}=9.726$

$\left|\pi_{\text {max }}\right|=12.043$
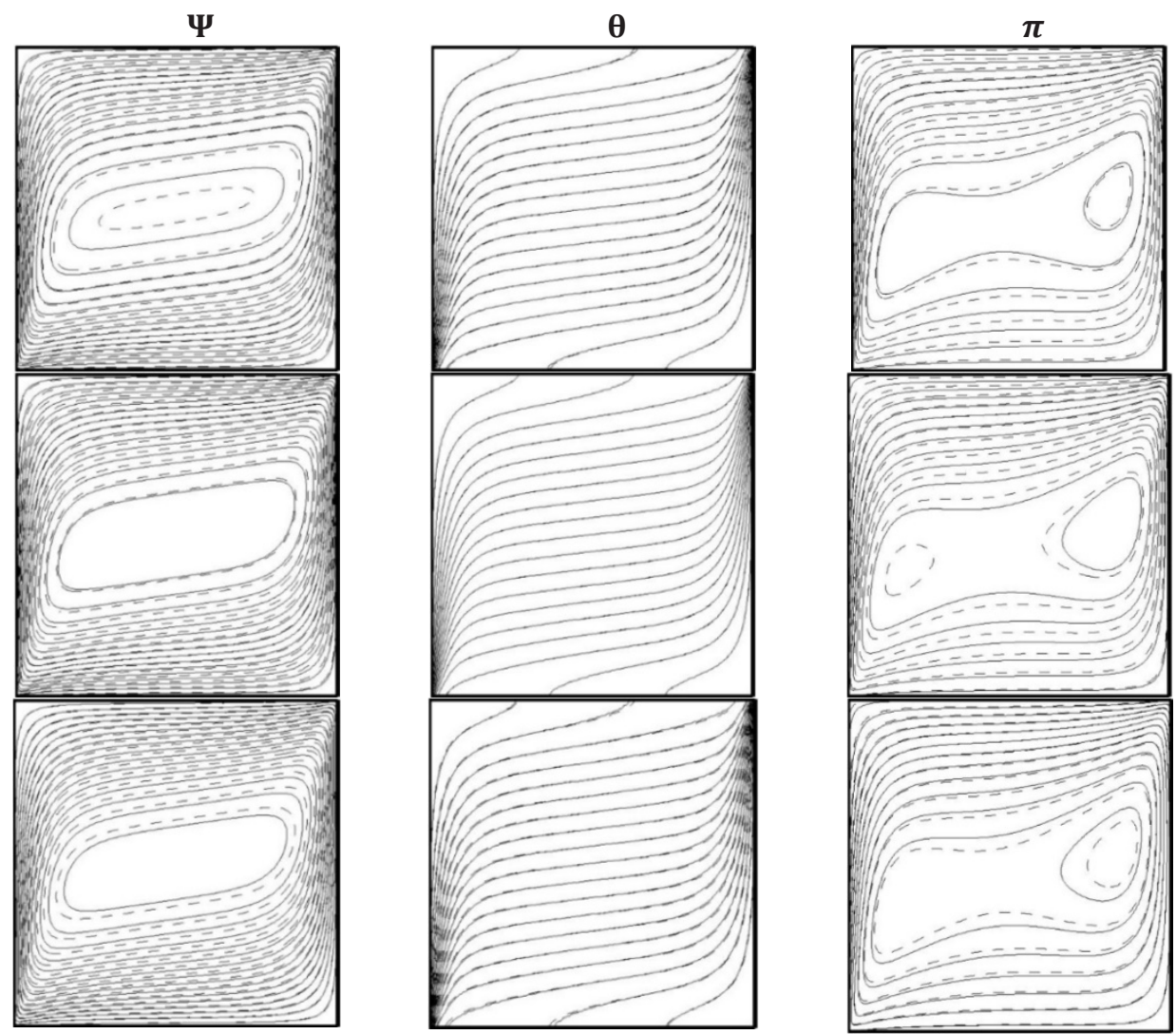

Figure 10. Distributions of streamlines, isotherms, and heatlines for different nanofluids

( $R a=1000, H a=1, \gamma=0, \phi=4 \%)$; dashed lines correspond to pure water with $\left|\Psi_{\max }\right|=14.962, \overline{N u}=9.357$, and $\left|\pi_{\max }\right|=11.516$

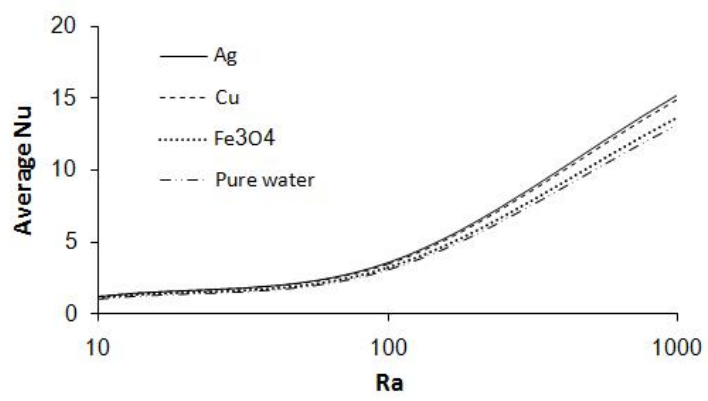

(a) $H a=0$

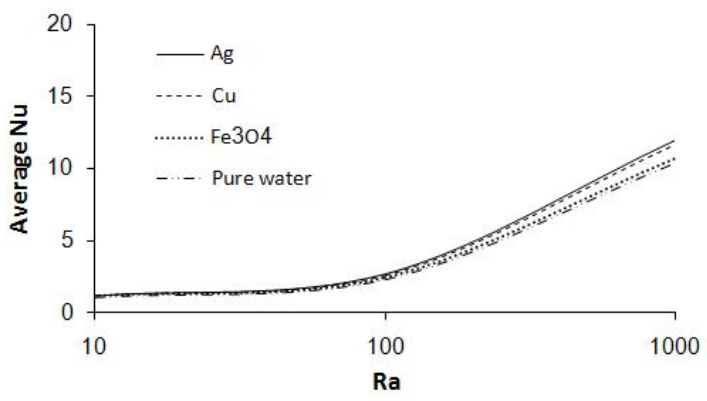

(c) $H a=1, \gamma=\pi / 4$

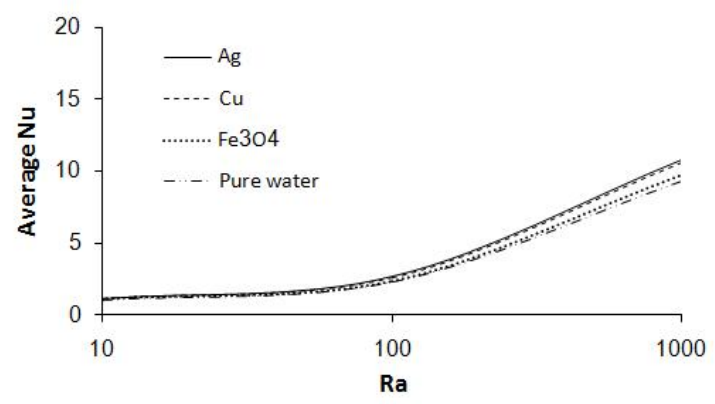

(b) $H a=1, \gamma=0$

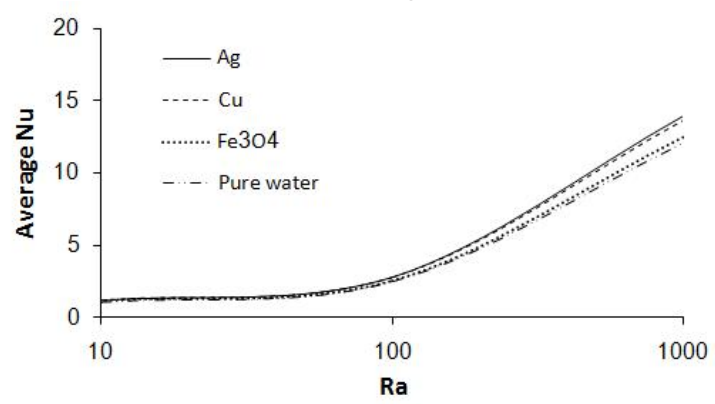

(d) $H a=1, \gamma=\pi / 2$

Figure 11. The average Nusselt number vs. the Rayleigh number for different nanofluids with $\phi=4 \%$ compared with pure water 


\section{CONCLUSION}

In this study, the heatline visualization technique was adopted to study the consequences of the magnetic field orientation on free convection of several water-based nanofluids in a square porous cavity. From our numerical results, the following major outcomes are listed:

- With increase in the magnitude of an externally applied magnetic field, the flow strength and the resulting heat transfer are deteriorated.

- Suppression effect of the horizontal magnetic field is more pronounced than that of the vertical or inclined ones.

- The consequences of the nanoparticles type and volume fraction on $\overline{N u}$ are more prominent at higher Rayleigh numbers.

- The Ag-water nanofluid has the utmost heat transfer among the current water-based nanofluids.

- Depending on the employed correlation for $\rho_{n f} \beta_{n f}$, one may predict increase or decrease in $\overline{N u}$ with the inclusion of the nanoparticles in the base fluid.

\section{NOMENCLATURE}

$B$

$C$

$\mathrm{Da}$

$\boldsymbol{g}$

$h$

$\mathrm{Ha}$

$k$

K

$L$

$\mathrm{Nu}$

$\overline{N u}$

$p$

$R a$

$T$

$u, v$

$x, y$

$X, Y$

Greek symbols

$\alpha$

$\beta$

$\gamma$

$\boldsymbol{\Theta}$

$\mu$

$\Pi$

$\rho$

$\sigma$

$\phi$

$\psi$

$\Psi$

Subscripts

bf

C

H

$n f$

$p$ magnetic field, $\mathrm{T}$

specific heat, $\mathrm{Jkg}^{-1} \mathrm{~K}^{-1}$

Darcy number

gravitational acceleration, $\mathrm{ms}^{-2}$

heat function, $\mathrm{Wm}^{-1}$

Hartmann number

thermal conductivity, $\mathrm{Wm}^{-1} \mathrm{~K}^{-1}$

medium permeability, $\mathrm{m}^{2}$

cavity length and height, $\mathrm{m}$

local Nusselt number

average Nusselt number

pressure, $\mathrm{Pa}$

Rayleigh number

temperature, $\mathrm{K}$

velocity components in $\mathrm{x}$ and $\mathrm{y}$ directions, respectively, $\mathrm{ms}^{-1}$

cartesian coordinates, $\mathrm{m}$

dimensionless coordinates

thermal diffusivity, $\mathrm{m}^{2} \mathrm{~s}^{-1}$

thermal expansion coefficient, $\mathrm{K}^{-1}$

magnetic field inclination angle

dimensionless temperature

dynamic viscosity, Pas

dimensionless heat function

density, $\mathrm{kgm}^{-3}$

electrical conductivity, $\Omega^{-1} \mathrm{~m}^{-1}$

nanoparticles fraction

stream function, $\mathrm{m}^{2} \mathrm{~s}^{-1}$

dimensionless stream function

base fluid

cold

hot

nanofluid

nanoparticle 


\section{REFERENCES}

[1] Alchaar S, Vasseur P, Bilgen E. Hydromagnetic natural convection in a tilted rectangular enclosure. Numer Heat Transfer, Part A 1995;27:107-127. doi:10.1080/10407789508913691.

[2] Bian W, Vasseur P, Bilgen E. Effect of an external magnetic field on buoyancy driven flow in a shallow porous cavity. Numer Heat Transfer, Part A 1996;29:625-638. doi:10.1080/1040778960891381.

[3] Bian W, Vasseur P, Bilgen E, Meng F. Effect of an electromagnetic field on natural convection in an inclined porous layer. Int J Heat Fluid Flow 1996;17:36-44. doi:10.1016/0142-727X(95)00070-7.

[4] Khanafer K, Chamkha AJ. Hydromagnetic natural convection from an inclined porous square enclosure with heat generation. Numer Heat Transfer, Part A 1998;33:891-910. doi:10.1080/10407789808913972.

[5] Mahmud S, Fraser RA. Magnetohydrodynamic free convection and entropy generation in a square cavity. Int J Heat Mass Transfer 2004;47:3245-3256. doi:10.1016/j.ijheatmasstransfer.2004.02.005.

[6] Grosan T, Revnic C, Pop I, Ingham DB. Magnetic field and internal heat generation effects on the free convection in a rectangular cavity filled with a porous medium. Int J Heat Mass Transfer 2009;52:1525-1533. doi:10.1016/j.ijheatmasstransfer.2016.08.025.

[7] Pekmen B, Tezer-Sezgin M. DRBEM solution of free convection in porous enclosures under the effect of a magnetic field. Int J Heat Mass Transfer 2013;56:454-468. doi:10.1016/j.ijheatmasstransfer.2012.09.019.

[8] Kumar V, Krishna Murthy SVSSNVG, Rathish Kumar BV. Influence of MHD forces on Bejan's heatlines and masslines in a doubly stratified fluid saturated Darcy porous enclosure in the presence of Soret and Dufour effects - A numerical study. Int J Heat Mass Transfer 2018;117:1041-1062. doi:10.1016/j.ijheatmasstransfer.2017.10.054.

[9] Geridönmez BP. Numerical simulation of natural convection in a porous cavity filled with ferrofluid in presence of magnetic source. J Thermal Eng 2018;4:1756-1769. doi:10.18186/journal-of-thermal-engineering.369169.

[10] Mahfoud B, Bendjaghloli A. Natural convection of a nanofluid in a conical container. J Thermal Eng 2018;4:1713-1723. doi:10.18186/journal-of-thermal-engineering.367407.

[11] Singh P, Sharma P, Gupta R, Wanchoo RK. Heat transfer characteristics of propylene glycol/water based magnesium oxide nanofluid flowing through straight tubes and helical coils. J Thermal Eng 2018;4:1737-1755. doi:10.18186/journal-of-thermal-engineering.369007.

[12] Zahmatkesh I, Naghedifar SA. Oscillatory mixed convection in jet impingement cooling of a horizontal surface immersed in a nanofluid-saturated porous medium. Numer Heat Transfer, Part A 2017;72:401-416. doi:10.1080/10407782.2017.1376961.

[13] Zahmatkesh I, Torshizi E. Scrutiny of unsteady flow and heat transfer in a backward-facing step under pulsating nanofluid blowing using the Eulerian-Eulerian approach. J Mech 2019;35:93-105. doi:10.1017/jmech.2017.73.

[14] Zahmatkesh I, Habibi MR. Natural and mixed convection of nanofluid in porous cavities: A critical analysis using the Buongiorno’s model. J Theor Appl Mech 2019;57:221-233. doi:10.15632/jtam-pl.57.1.221.

[15] Koopaee MK, Jelodari I. Numerical investigation of magnetic field inclination angle on transient natural convection in an enclosure filled with nanofluid, Engineering Computations 2014;31:1342-1360. doi:10.1108/EC-12-2012-0320.

[16] Koopaee MK, Omidvar A, Jelodari I. Numerical study on the steady-state heat transfer rate of nanofluid filled within square cavity in the presence of oriented magnetic field. Proc Inst Mech Eng, Part C: J Mech Eng Sci 2014;228:1348-1362. doi:10.1177/0954406213507860.

[17] Akinshilo A, Ilegbusi AO. Investigation of Lorentz force effect on steady nanofluid flow and heat transfer through parallel plates. J Thermal Eng 2019;5:482-497. doi:10.18186/thermal.625919.

[18] Malik S, Nayak AK. MHD convection and entropy generation of nanofluid in a porous enclosure with sinusoidal heating. Int J Heat Mass Transfer 2017;111:329-345. doi:10.1016/j.ijheatmasstransfer.2017.03.123.

[19] Mansour MA, Ahmed SE, Chamkha A. Entropy generation optimization for MHD natural convection of a nanofluid in porous media-filled enclosure with active parts and viscous dissipation. Int J Numer Methods Heat Fluid Flow 2017;27:379-399. doi:10.1108/HFF-10-2015-0408.

[20] Sheikholeslami M, Rokni HB. Magnetohydrodynamic CuO-water nanofluid in a porous complex-shaped enclosure. J Thermal Sci Eng Appl 2017;9;041007. doi:10.1115/1.4035973.

[21] Rashad AM, Rashidi MM, Lorenzini G, Ahmed SE, Aly AM. Magnetic field and internal heat generation effects on the free convection in a rectangular cavity filled with a porous medium saturated with $\mathrm{Cu}$-water nanofluid. Int J Heat Mass Transfer 2017;104:878-889. doi:10.1016/j.ijheatmasstransfer.2016.08.025.

[22] Rashad AM, Armaghani T, Chamkha AJ, Mansour MA. Entropy generation and MHD natural convection of a nanofluid in an inclined square porous cavity: Effects of a heat sink and source size and location. Chin J Phys 2018;56:193-211. doi:10.1016/j.cjph.2017.11.026. 
[23] Balla CS, Kishan N, Gorla RSR, Gireesha BJ. MHD boundary layer flow and heat transfer in an inclined porous square cavity filled with nanofluids. Ain Shams Eng J 2017;8:237-254. doi:10.1016/j.asej.2016.02.010.

[24] Zahmatkesh I, Shandiz MRH. Optimum constituents for MHD heat transfer of nanofluids within porous cavities: A Taguchi analysis in natural and mixed convection configurations, J Thermal Anal Calorim 2019;138:1669-1681. doi:10.1007/s10973-019-08191-y.

[25] Kimura S, Bejan A. The "heatline” visualization of convective heat transfer. J Heat Transfer 1983;105:916-919. doi:10.1115/1.3245684.

[26] Saleh H, Hashim I. Heatline visualization of natural convection in an inclined square porous enclosure with sinusoidal boundary conditions. J Porous Med 2013;16:875-885. doi:10.1615/JPorMedia.v16.i10.10.

[27] Saleh H, Hashim I. Heatline visualization of conjugate heat transfer in square porous enclosure. J Porous Med 2013;16:1119-1132. doi:10.1615/JPorMedia.v16.i12.50.

[28] Zahmatkesh I. Heatline visualization for buoyancy-driven flow inside a nanofluid-saturated porous enclosure. Jordan J Mech Indust Eng 2015;9:149-157.

[29] Alsabery AI, Chamkha AJ, Hussain SH, Saleh H, Hashim I. Heatline visualization of natural convection in a trapezoidal cavity partly filled with nanofluid porous layer and partly with non-Newtonian fluid layer. Adv Powder Technol 2015;26:1230-1244. doi:10.1016/j.apt.2015.06.005.

[30] Hussain S.H. Analysis of heatlines and entropy generation during double-diffusive MHD natural convection within a tilted sinusoidal corrugated porous enclosure. Eng Sci Technol 2016;19:926-945. doi:10.1016/j.jestch.2015.12.001.

[31] Bondareva NS, Sheremet MA, Abu-Hamdeh N. Heatline visualization of MHD natural convection in an inclined wavy open porous cavity filled with a nanofluid with a local heater. Int $\mathrm{J}$ Heat Mass Transfer 2016;99:872-881. doi:10.1016/j.ijheatmasstransfer.2016.04.055.

[32] Maxwell JA. Treatise on Electricity and Magnetism. Cambridge: Oxford University Press; 1904. doi:10.1017/CBO9780511709340.

[33] Brinkman HC. The viscosity of concentrated suspensions and solutions. J Chem Phys 1952;20:571-581. doi:10.1063/1.1700493.

[34] Bourantas GC, Skouras ED, Loukopoulos VC, Burganos VN. Heat transfer and natural convection of nanofluids in porous media. Eur J Mech-B/Fluids 2014;43:45-56. doi:10.1016/j.euromechflu.2013.06.013.

[35] Zahmatkesh I. On the suitability of the volume-averaging approximation for the description of thermal expansion coefficient of nanofluids. Proc Inst Mech Eng, Part C: J Mech Eng Sci 2015;229:2835-2841. doi:10.1177/0954406214563735.

[36] Sun Q, Pop I. Free convection in a triangle cavity filled with a porous medium saturated with nanofluids with flush mounted heater on the wall. International Journal of Thermal Sciences 2011;50:2141-2153. doi:10.1016/j.ijthermalsci.2011.06.005.

[37] Chamkha AJ, Ismael MA. Conjugate heat transfer in a porous cavity filled with nanofluids and heated by a triangular thick wall. Int J Thermal Sci 2013;67:135-151. doi:10.1016/j.ijthermalsci.2012.12.002. 